offered regular reviews for adults with $\mathrm{CD}$, detailed in the table.

Conclusions Meeting national guidance is a key driver for provision and content of reviews for adults with CD. This study provides a baseline for comparison to how the services have changed during the pandemic and beyond. There is urgent need to evaluate the acceptability of different formats and include the voice of people with CD in the design of services.

\section{PTH-12 CATHETER-RELATED INFECTION RATES IN PATIENTS ON CUSTOMIZED HOME PARENTERAL NUTRITION COMPARED TO MULTI-CHAMBER BAGS}

\footnotetext{
1,2Benjamin Crooks*, 'Simon Harrison, ${ }^{1}$ Graham Millward, ${ }^{1}$ Kirsty Hall, ${ }^{1}$ Michael Taylor, ${ }^{1}$ Kirstine Farrer, ${ }^{1}$ Arun Abraham, ${ }^{1}$ Antje Teubner, ${ }^{1,2}$ Simon Lal. ${ }^{1}$ Intestinal Failure Unit, Salford Royal NHS Foundation Trust, Salford, UK; ${ }^{2}$ The University of Manchester, Manchester, UK
}

\subsection{6/gutjnl-2021-BSG.291}

Introduction The risk of blood stream infections may be increased in hospitalized patients receiving ready-made multichamber parenteral nutrition bags (MCB) compared to customized parenteral nutrition (PN); however, as highlighted in recent international guidelines, there are no comparable data relating to home $\mathrm{PN}$ (HPN).

Methods Data from a prospectively maintained database were analysed to compare incidence rates of catheter-related blood stream infections (CRBSI) between patients receiving customized HPN compared to MCB HPN at a national UK referral centre between May 2018 and August 2020. Two patient cohorts were included:

1. Patients newly commenced on HPN (customized and MCB) for a 14 month period from July 2019.

2. Patients who were switched from customized to $\mathrm{MCB}$ HPN in July 2019.

Results Sixty patients with chronic intestinal failure were commenced on MCB and 45 on customized HPN for a total of 5914 and 7641 catheter days respectively. No difference in CRBSI incidence was found $(0.51 / 1000$ catheter days for MCB, 0.39/1000 catheter days for customized HPN; incidence rate ratio $1.29,95 \%$ CI $0.26-6.37$ )

Eighteen patients were switched from customized HPN to MCB HPN. The study period covered 7401 catheter days on customized HPN and 4834 days on MCB. No significant change was noted in the CRBSI rates following this switch $(0.27 / 1000$ catheter days on customized HPN versus 0.21 / 1000 catheter days on $\mathrm{MCB}$; incidence rate ratio $1.31,95 \%$ CI 0.12-14.3).

Conclusions The use of MCB for HPN patients is not associated with an increased risk of CRBSI. This study will inform international guidelines and provide reassurance for the continued, safe use of MCB HPN.

\section{PTH-13 ONGOING PH TESTING OF NASOGASTRIC TUBES: A REVIEW OF HOSPITAL INCIDENT REPORTS IN ADULTS}

\footnotetext{
${ }^{1}$ Kate Glen*, ${ }^{1}$ Merrilyn Banks, ${ }^{2}$ Christine Elizabeth Weekes, ${ }^{1}$ Mary Hannan-Jones. ${ }^{1}$ Queensland University of Technology, Brisbane, Australia; ' ${ }^{2}$ Guy's and St Thomas' Hospitals NHS Foundation Trust, London, UK
}

Introduction It is unknown if $\mathrm{pH}$ testing a nasogastric tube (NGT) before every use reduces the risk of adverse outcomes as it is based on expert opinion ${ }^{(1,2)}$. Adverse outcomes such as never events are rare, 95 in 4.5 year period across England, with 790,000 NGTs supplied annually ${ }^{(1)}$ making this difficult to observe such incidents in cohort studies. This review of hospital NGT incident data aims to identify incidents related to ongoing $\mathrm{pH}$ testing, at a central London hospital which orders approximately 5,000 NGTs annually.

Methods The hospital's Risk Systems Manager searched all incident data from 1-Jan-2014 to 31-Dec-2019 for [Nasogastric OR NG] AND tube. Anonymised short incident descriptions were shared with the authors who compared the incidents to inclusion/exclusion criteria (see figure 1). More detailed incident records were requested for included incidents and compared against the criteria. Incidents were analysed using descriptive statistics. This review considered a service evaluation, so no ethical approval required.

Results See figure 1 for results. The initial search returned 352 incidents. Further description obtained for 22 incidents,

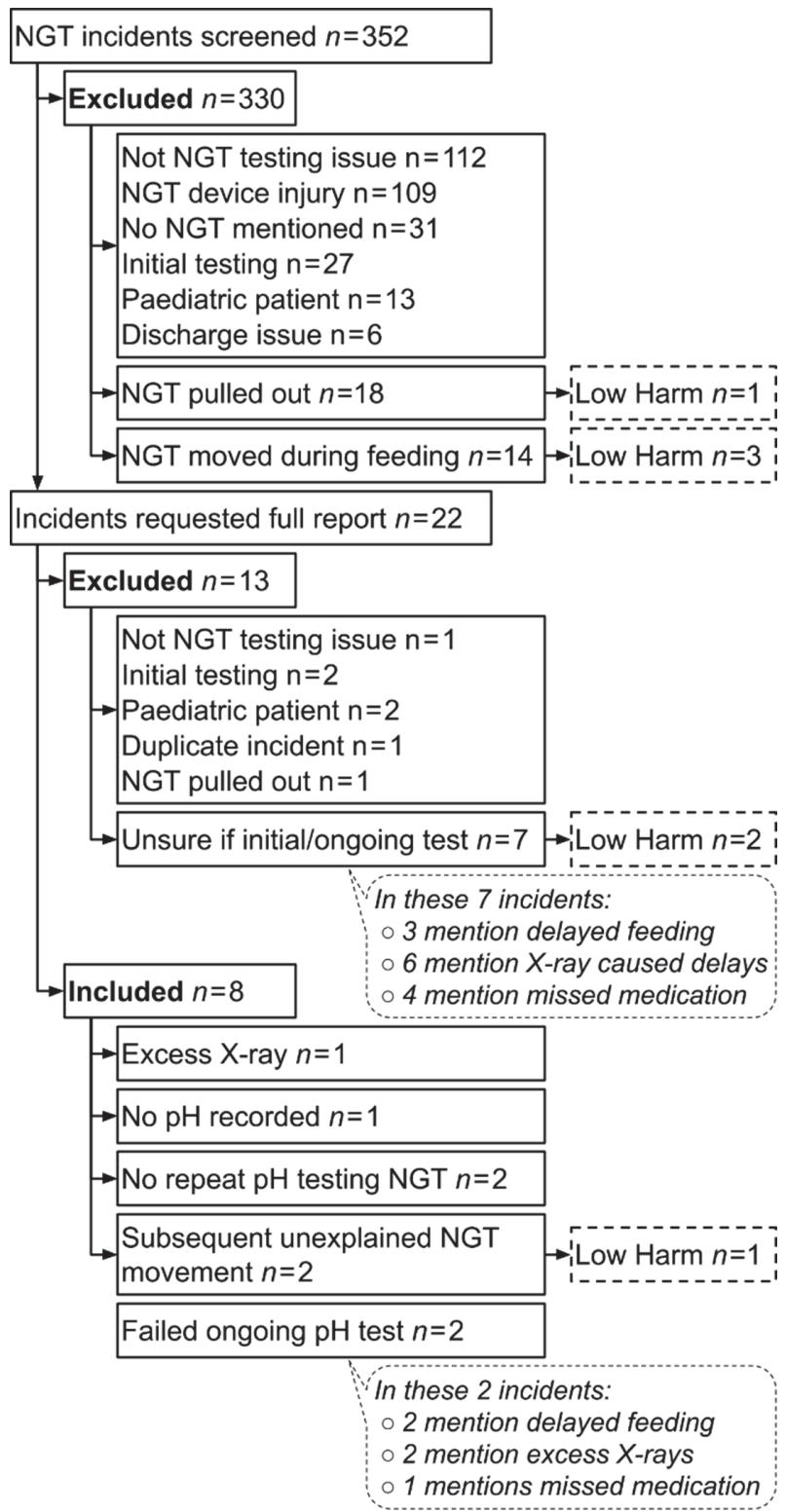

Abstract PTH-13 Figure 1 Classification tree of incidents 